\title{
Blood Pressure Monitoring
}

1.3 Reproducibility of Dipping/Non Dipping Pattern in Untreated Essential Hypertensives: Impact of Gender and Age

F. Negri, ${ }^{1}$ C. Cuspidi,,2 S. Meani, ${ }^{3}$ C. Valerio,3 C. Sala, 3 V. Fusi, 3 A. Esposito, 1

M. Maisaidi,3 A. Zanchetti,4 G. Mancia2

(1) Policlinico di Monza, Mo Monza; (2) Università Milano-Bicocca, Policlinico di

Monza, Mo Monza; (3) Istituto Medicina Cardiovascolare Osp. Maggiore

Policlinico IRCCS, Mi Milano; (4) Centro interuniversitario Fisiol. Cli. e Ipertensione

Università Milano, Milano, It Italy

Objective: We aimed to evaluate the intrasubject short-term reproducibility of nocturnal blood pressure (BP) patterns (dipping/non-dipping) in essential hypertensive patients in relation to age ( $>50$ years) and gender.

Methods: A total of 619 never-treated essential grade 1 and 2 hypertensives ( 383 men, 236 women) underwent the following procedures: 1) repeated clinic BP measurements, 2) routine examinations, 3 ) ambulatory BP monitoring (ABPM) over two 24-h periods within 4 weeks. Dipping pattern was defined as a $10 \%$, or more, reduction in average SBP/DBP at night compared to daytime values. Results: Of the 407 patients showing a dipping pattern during the first ABPM period, $329(80.1 \%)$ had the same pattern during the second ABPM recording. Of the 211 patients with a non-dipping pattern during the first ABPM period, $140(65.9 \%)$ confirmed the same pattern during the second ABM period. Overall, 149 patients $(24.1 \%)$ changed their initial nocturnal pattern at the second ABPM recording, without significant age and gender-related differences. Lower reproducibility rates of the non-dipping as compared to the dipping pattern were found in the whole population as well as in men regardless of age and in younger but not in older women.

Conclusions: These findings indicate that nocturnal BP patterns have a limited short-term reproducibility in the whole study population as well as in different age and gender subgroups. As variability of nocturnal BP patterns is not predicted by easy available clinical data, such as gender and age, a reliable classification of patients according to circadian BP patterns should be obtained by repeating ABPM. 